M. ET $M^{\text {me }}$ MOREAU. - SUR UNE LIANE HERMAPHRODITE DE HOUBLON.

\title{
Sur une liane hermaphrodite de Houblon
}

\author{
PAR M. ET M me FERNAND MOREAU.
}

M. Michel Hermann ayant remarqué dans sa houblonnière de Dunzenheim (Bas-Rhin) une tige de Houblon que le Journal Agricole d'Alsace et de Lorraine du 17 septembre 1921 indiquait comme constituant un cas d'hermaphroditisme, nous avons prié M. Hermann de vouloir bien nous envoyer un échantillon de cette anomalie, ce qu'il a fait avec un empressement dont nous le remercions. A plusieurs reprises déjà, des lianes hermaphrodites de Houblon ont été signalées, apparues dans des plantations (Brunotte ${ }^{1}$, Winge ${ }^{2}$ ) ou provoquées dans des cultures expérimentales (Tournois ${ }^{3}$ ); quelques-unes d'entre elles ont été l'objet de recherches cytologiques (Winge). Bien que les échantillons dont nous avons disposé, examinés seulement plusieurs semaines après leur récolte et déjà desséchés, ne nous aient pas permis de faire une étude complète de la liane anormale de Dunzenheim, nous pensons qu'il est intéressant d'en faire connaître les caractères principaux.

Cette liane, d'après les quelques rameaux que nous possédons, frappe par la vigueur de sa végétation : elle porte de nombreux cônes de grande taille et présentant des feuilles bien développées parmi les bractées, comme il arrive souvent chez les cônes à végétation exubérante. Un examen moins sommaire montre que certains rameaux portent des inflorescences grêles, richement ramifiées, ayant le port des panicules mâles du Houblon, tandis que d'autres présentent des grappes lâches d'inflorescences courtes, trapues, très feuillues, ressemblant à de petits cônes femelles. Nous avons donc eu à examiner des cônes, des inflorescences andromorphes et des inflorescences gynomorphes.

1. Brunotte (C.), Sur une liane de Houblon 'Humulus Lupulus) hermaphrodite (Rev. gén. de Bot., XVII, p. 109-208, 1905).

2. WINGE $(0)$, The pollination and fertilization process in Humulus Lupulus p. 26, 1914). japonicus Sieb. et Succ. (C. R. Trav. du Lab. de Carsberg, II,

3. Tournois (J.), Études sur la sexualité du Houblon (Ann. Sc. nat., Bot., ${ }^{9}$ sér., XIX, p. 49-191, 1914). 


\section{I. - LES GÔNES}

Les cônes, ou inflorescences femelles normales, constituent la forme d'inflorescence qui domine dans la liane hermaphrodite de Dunzenheim; cependant nous n'avons pas reçu de grappes florifères qui en soient exclusivement formées. Leur taille est élevée, leur longueur pouvant dépasser 4 centimètres; ce sont des cônes « feuillus », surtout à leur base, et portant plus haut, depuis leur milieu jusque près de leur extrémité, à chacun de leurs nœuds, un appendice filiforme qui représente une feuille rudimentaire; la plupart sont graineux et cette circonstance accroit encore leurs dimensions et celles de leurs diverses parties ${ }^{1}$; toutefois, même en dehors de toute fécondation, le développement des cônes est plus grand qu'il l'est en général pour les Houblons de la même région d'Alsace. Ainsi, la moyenne de la longueur et la moyenne de la largeur des bractéoles ${ }^{2}$ sans graine pour 12 houblons de la région de Strasbourg sont respectivement $13,9 \mathrm{~mm}$. et $7,2 \mathrm{~mm}$; ; elles sont respectivement de $17,1 \mathrm{~mm}$. et $8,5 \mathrm{~mm}$. pour les cônes de la liane hermaphrodite de Dunzenheim. Ces nombres deviennent $2.5 \mathrm{~mm}$. et $13,4 \mathrm{~mm}$. pour les bractéoles à graine dans le dernier cas, alors que $18,3 \mathrm{~mm}$. et $10,9 \mathrm{~mm}$. sont les dimensions moyennes des bractéoles graineuses des Houblons d'Alsace.

Les caractères qui précèdent, et qui témoignent d'une exubérance de la végétation, sont particulièrement accusés dans les cônes des extrémités des grappes.

\section{II. - LES INFLORESGENGES ANDROMORPHES}

Certains rameaux secondaires de branches portant des cônes, se développent en panicules ayant l'apparence générale des.

1. MOREAU (F. et $\mathrm{M}^{\mathrm{me}}$ ), Recherches sur le Houblon, p. 34. Lons-le-Saunier, Declume, 1922.

2. Les descriptions qui vont suivre présupposent la connaissance de la structure du cône femelle; nous désignons sous le nom de bractées les pièces foliacées du cône insérées par paires aux divers étages de son rachis. et sous celui de bractéoles, les pièces, généralement au nombre de quatre par étage, qui entourent à leur base les fleurs femelles ou les graines [C. Moreau (F. et $\mathbf{N}^{\text {me }} \mathbf{F}$.), Etude morphologique des inflorescences du Houblon (Humulus Lupulus L. (Bull. Soc. bot. Fr., LXIX, p. 527, 1922]. 
M. ET M ${ }^{\text {me }}$ yOREAU. - SUR UNE LIANE HERMAPHRODITE DE HOUBLON.

panicules des pieds mâles du Houblon. De telles panicules se trouvent aussi, au nombre de deux, à la base des rameaux terminés par un cône.

Ce sont des inflorescences grêles, lâches et très fournies en fleurs grâce à leur riche ramification. A la base des rameaux de ces inflorescences andromorphes sont des feuilles représentées chacune par deux stipules; ces stipules sont plus développées que celles des panicules des pieds mâles; quelques-unes d'entre elles atteignent 5 à 6 millimètres de long et 3 à 4 millimètres de large, elles offrent alors la forme, la consistance, la nervation, la couleur, en un mot l'aspect général des bractées des cònes dont elles ne diffèrent que par leur taille plus petite.

Les fleurs de ces panicules sont souvent du type mâle, avec un périanthe sépaloïde, formé de 5 pièces, et 5 étamines. En outre, on trouve parmi elles quelques fleurs hermaphrodites, comprenant un périanthe de 3,4 ou 5 pétales, des étamines en nombre variant entre les mêmes limites et un pistil laissant voir 1, 2 ou 3 styles. Les étamines de ces inflorescences sont sans pollen visible dans les échantillons étudiés, mais, examinées après un mois environ de dessiccation, elles paraissent vidées de leur pollen. Il est possible que l'abondance des graines des cônes femelles soit due à l'action du pollen des inflorescences andromorphes voisines.

\section{III. - LES INFLORESGENGES GYNOMORPHES}

Les inflorescences gynomorphes sont encore des inflorescences hermaphrodites mais qui, par le développement des bractées et des bractéoles. rappellent les cônes femelles.

Elles sont toujours de petite taille; elles forment souvent un court rameau secondaire d'une branche qui se termine par un cône femelle, mais elles peuvent aussi occuper d'autres positions. Elles sont peu ramifiées; leur aspect est celui de tout petits cônes ou de panicules ramifiées de petits cônes très réduits.

Chacun d'eux comprend le plus souvent deux bractées, deux bractéoles avec des fleurs femelles réduites et se termine par T. LXIX. 
une fleur hermaphrodite aux étamines en nombre souvent déficient (trois par exemple, au lieu de cinq), aux sépales inégalement développés et portant des glandes à lupuline sur leur face externe. Rarement ces inflorescences gynomorphes réduites comprennent plus d'un étage de bractées ou de bractéoles; parfois on trouve, correspondant à une paire de bractées, quatre bractéoles.

Entre le cône femelle typique et la panicule typique des pieds de Houblon mâle se trouvent donc des inflorescences hermaphrodites plus ou moins complexes, procédant des précédentes entre lesquelles elles sont intermédiaires.

En dehors de l'intérêt théorique qu'offre la rencontre sur une même liane, réputée femelle, d'inflorescences hermaphrodites, un autre intérêt s'attache à leur observation. Quand on veut opérer des croisements chez le Houblon, le plus généralement on part d'un matériel dont les qualités paternelles sont ineonnues; on sait à quelle sorte appartient la plante femelle porte-graines, mais on ignore à quelle sorte appartient la plante mâle qui a fourni le pollen; on part ordinairement d'une graine recueillie dans un cône fécondé, et on ne sait rien du progéniteur mâle, un houblon sauvage ou subspontané d'une haie voisine et sans généalogie définie. Il n'en est pas de même si on dispose de rameaux hermaphrodites d'un Houblon appartenant à un clone ${ }^{1}$ connu; il est alors possible, s'ils.produisent un pollen fécondant, de se servir de ce pollen pour féconder des fleurs femelles du même clone ou de la même sorte ou de sortes ou de clones différents. Attirant l'attention des botanistes et des planteurs sur l'intérêt à ce point de vue des rameaux hermaphrodites du Houblon cultivé, nous serions heureux qu'ils veuillent bien nous faire connaitre, en vue d'expériences d'hybridation, les cas d'hermaphroditisme de Houblon - probablement moins rares qu'on le croit - qu'ils auraient l'occasion de rencontrer.

1. Nous introduisons ici le mot clone, déjà utilisé en anglais, pour désigner l'ensemble des individus obtenus par voie asexsuelle à partir d'un individu primitif. Toutes les pommes de terre obtenues par plantation de tubercules à partir d'un tubercule unique, tous les Houblons issus par bouturage répété d'un premier Houblon forment un clone. 


\section{$2 \mathrm{BHL}$ Biodiversity Heritage Library}

Moreau, Fernand and Moreau, Valentine. 1922. "Sur une liane hermaphrodite de Houblon." Bulletin de la Société botanique de France 69, 591-594. https://doi.org/10.1080/00378941.1922.10833483.

View This Item Online: https://www.biodiversitylibrary.org/item/93160

DOI: https://doi.org/10.1080/00378941.1922.10833483

Permalink: https://www.biodiversitylibrary.org/partpdf/161288

\section{Holding Institution}

Missouri Botanical Garden, Peter H. Raven Library

\section{Sponsored by}

Missouri Botanical Garden

\section{Copyright \& Reuse}

Copyright Status: Public domain. The BHL considers that this work is no longer under copyright protection.

This document was created from content at the Biodiversity Heritage Library, the world's largest open access digital library for biodiversity literature and archives. Visit BHL at https://www.biodiversitylibrary.org. 